\title{
Anhang C: Weitere nützliche Formeln
}

\section{C.1 Matrizenrechnung}

$2 \times 2$ Matrixinversion:

$$
\left(\begin{array}{ll}
a & b \\
c & d
\end{array}\right)^{-1}=\frac{1}{a d-b c}\left(\begin{array}{cc}
d & -b \\
-c & a
\end{array}\right)
$$

Symmetrische $3 \times 3$ Matrixinversion:

$$
\left(\begin{array}{lll}
x & c & b \\
c & y & a \\
b & a & z
\end{array}\right)^{-1}=\frac{1}{D}\left(\begin{array}{lll}
z y-a^{2} & a b-c z & c a-b y \\
a b-c z & x z-b^{2} & c b-a x \\
c a-b y & c b-a x & x y-c^{2}
\end{array}\right)
$$

mit der Determinante $D=2 a b c+x y z-a^{2} x-b^{2} y-c^{2} z$

\section{C.2 Endliche und unendliche Summen}

Geometrische Reihe

$$
\begin{gathered}
\sum_{i=0}^{n} q^{i}=\frac{1-q^{n+1}}{1-q} \\
\sum_{i=1}^{n} i q^{i-1}=\frac{\mathrm{d}}{\mathrm{d} q} \sum_{i=1}^{n} q^{i}=\frac{1+n q^{n+1}-(n+1) q^{n}}{(1-q)^{2}} \\
\sum_{i=k}^{n} i(i-1) \cdots(i-k+1) q^{i-k}=\frac{\mathrm{d}^{k}}{\mathrm{~d} q^{k}} \sum_{i=1}^{n} q^{i}
\end{gathered}
$$

Für $n=\infty$ und $|q|<1$ :

$$
\begin{gathered}
\sum_{i=0}^{\infty} q^{i}=\frac{1}{1-q} \\
\sum_{i=0}^{\infty}(i+1) q^{i}=\frac{1}{(1-q)^{2}} \\
\sum_{i=0}^{\infty}(i+1)(i+2) \cdots(i+k) q^{i}=\sum_{i=0}^{\infty} i(i-1)(i-2) \cdots(i-k+1) q^{i-k}=\frac{k !}{(1-q)^{k+1}}
\end{gathered}
$$

Die zweite Summe hat erst ab $i=k$ von 0 verschiedene Summanden. Diese Beziehung entspricht der Taylor-Entwicklung der rechten Seite um $q=0$. 